\title{
The significance of differences in fatty acid metabolism between obese and non-obese patients with non-alcoholic fatty liver disease
}

\author{
MAKOTO NAKAMUTA ${ }^{1,2}$, MOTOYUKI KOHJIMA ${ }^{3}$, NOBITO HIGUCHI ${ }^{3}$, \\ MASAKI KATO $^{3}$, KAZUHIRO KOTOH ${ }^{3}$, TSUYOSHI YOSHIMOTO ${ }^{1,2}$, \\ MASAYOSHI YADA ${ }^{3}$, RYOKO YADA $^{2}$, RYOSUKE TAKEMOTO ${ }^{1,2}$, \\ KUNITAKA FUKUIZUMI ${ }^{1,2}$, NAOHIKO HARADA ${ }^{1,2}$, AKINOBU TAKETOMI $^{4}$, \\ YOSHIHIKO MAEHARA $^{4}$, MANABU NAKASHIMA ${ }^{5}$ and MUNECHIKA ENJOJI ${ }^{5}$ \\ ${ }^{1}$ Department of Gastroenterology, ${ }^{2}$ Clinical Research Center, Kyushu Medical Center, National Hospital \\ Organization; ${ }^{3}$ Departments of Medicine and Bioregulatory Science, ${ }^{4}$ Surgery and Medical Science, \\ Department of Graduate School of Medical Sciences, Kyushu University; ${ }^{5}$ Department of Clinical \\ Pharmacology, Faculty of Pharmaceutical Sciences, Fukuoka University, Fukuoka, Japan
}

Received July 7, 2008; Accepted August 26, 2008

DOI: 10.3892/ijmm_00000070

\begin{abstract}
Non-alcoholic fatty liver disease (NAFLD) is considered to be associated with metabolic syndrome; however, a number of NAFLD patients are not obese. To explore any differences in lipid metabolism between obese and non-obese patients, we determined the expression of fatty acid metabolism-related genes. Expression levels of target genes were quantified by real-time PCR using liver biopsy samples from NAFLD patients and normal controls. Serum adipocytokine levels were also determined. The expression of
\end{abstract}

Correspondence to: Dr Munechika Enjoji, Department of Clinical Pharmacology, Faculty of Pharmaceutical Sciences, Fukuoka University, 8-19-1 Nanakuma, Jonan-ku, Fukuoka 814-0180, Japan E-mail: enjoji@adm.fukuoka-u.ac.jp

Abbreviations: NAFLD, non-alcoholic fatty liver disease; ACC, acetyl-CoA carboxylase; FAS, fatty acid synthase; SREBP-1c, sterol regulatory element-binding protein-1c; ADRP, adipose differentiation-related protein; CPT1a, carnitine palmitoyltransferase 1a; LCAD, long-chain acyl-CoA dehydrogenase; HADH $\alpha$, long-chain L-3-hydroxyacyl-coenzyme A dehydrogenase $\alpha$; UCP2, uncoupling protein 2; ACOX, straight-chain acyl-CoA oxidase; BOX, branched-chain acyl-CoA oxidase; CYP, cytochrome P450; PPAR, peroxisome proliferator-activated receptor; DGAT1, diacylglycerol O-acyltransferase 1; HSL, hormone sensitive lipase; SOD, superoxide dismutase; GSS, glutathione synthetase; ROS, reactive oxygen species; BMI, body mass index; SOCS-3, suppressor of cytokine signaling-3

Key words: non-alcoholic fatty liver disease, fatty acid, oxidation, reactive oxygen species, leptin genes related to fatty acid synthesis and uptake was generally up-regulated in NAFLD patients; however, no significant difference was seen between obese and non-obese groups. Most of the genes tested related to fatty acid and reactive oxygen species (ROS) elimination, were overexpressed in NAFLD and the levels were significantly higher in non-obese patients. As an exception, peroxisome proliferator-activated receptor $\alpha$ expression was suppressed in NAFLD and the levels were lower in the obese group. Triglyceride synthesisrelated genes were up-regulated and lipolytic enzymes were decreased in NAFLD, but there was no significant difference between the obese and non-obese groups. In NAFLD, increased de novo synthesis and uptake of fatty acids led to further hepatocyte accumulation of fatty acids. The upregulation of fatty acid oxidation and the antioxidant pathway and the suppression of lipolysis seemed to be involved in this process. Expression of genes related to fatty acid oxidation and ROS elimination were higher in the non-obese group than in the obese group, which contributes to the trend of more severe liver injury, insulin resistance and steatosis in obese patients.

\section{Introduction}

Non-alcoholic fatty liver disease (NAFLD), which is characterized by triglyceride accumulation in hepatocytes (hepatic steatosis), is one of the most common hepatic diseases and its prevalence has markedly increased (1-3). More than $10 \%$ of NAFLD patients progress to a severe form, with hepatitis and fibrosis, non-alcoholic steatohepatitis (NASH), and in more severe cases, cirrhosis, hepatic failure and hepatocellular carcinoma (3-5). Therefore, it is critical to understand lipid metabolism, particularly fatty acid metabolism in NAFLD.

Fatty acids in the liver are derived from de novo synthesis and uptake of plasma free fatty acids. Up-regulation of synthesis and/or uptake can result in fatty acid accumulation. 
In turn, fatty acids in hepatocytes are metabolized by two pathways: i) oxidation to generate ATP in the mitochondria microsomes and peroxisomes and ii) esterification to produce triglycerides, which are either incorporated into lipoproteins for export or stored as a lipid droplet within the hepatocytes. Dysfunction of these pathways can lead to hepatic steatosis. We have previously estimated the role of these pathways in NAFLD patients by evaluating the expression of genes related to fatty acid metabolism (6-10). We found that all of the pathways investigated, namely de novo synthesis, oxidation of fatty acids, reactive oxygen species (ROS) elimination and triglyceride production, are significantly up-regulated in NAFLD. Therefore, dysregulation of de novo synthesis is thought to be a problem in NAFLD, that is to say, fatty acid synthesis was up-regulated despite lipid accumulation in hepatocytes.

NAFLD is often associated with obesity and/or insulin resistance and is considered to be closely implicated in metabolic syndrome; however, we often encounter non-obese NAFLD patients. In this study, using biopsy samples from obese and non-obese NAFLD and healthy livers, we compared hepatocyte fatty acid metabolism between obese and non-obese NAFLD patients by determining the expression of genes involved in de novo synthesis, uptake, oxidation, antioxidant pathways and triglyceride synthesis and catalysis.

\section{Patients and methods}

Sample tissue was obtained by liver biopsy from 25 patients with histologically diagnosed NAFLD, including four patients with NASH, who were admitted to the Kyushu University Hospital between 2004 and 2006. Patients were divided into two groups; non-obese (body mass index: BMI $<25 \mathrm{~kg} / \mathrm{m}^{2}$ ) and obese $\left(\mathrm{BMI}>25 \mathrm{~kg} / \mathrm{m}^{2}\right)$. The background and clinical characteristics of the patients with NAFLD are shown in Table I. As a control, healthy liver was also obtained by biopsy from 10 liver transplantation donors whose liver function test and histological findings were completely normal. Real-time RT-PCR was performed as previously reported (6).

Total RNA was prepared with TRIzol reagent (Invitrogen, Carlsbad, CA, USA) and cDNA was synthesized from $1.0 \mu \mathrm{g}$ RNA with GeneAmp ${ }^{\mathrm{TM}}$ RNA PCR (Applied Biosystems, Branchburg, NJ, USA) using random hexamers. PCR was performed using LightCycler-FastStart DNA Master SYBRGreen 1 (Roche, Tokyo, Japan) according to the manufacturer's instruction. The reaction mixture $(20 \mu 1)$ contained Light Cycler-FastStart DNA Master SYBR-Green 1, $4 \mathrm{mM} \mathrm{MgCl}_{2}$, $0.5 \mu \mathrm{M}$ of the upstream and downstream PCR primers and $2 \mathrm{ml}$ of the first-strand cDNA as a template. The primers for target genes have been described previously (6-8).

To adjust for variations in the reactions, all PCRs were normalized against $\beta$-actin expression. The expression levels of the tested genes are shown as a percentage of NAFLD against normal control. All results are shown as the mean \pm SD. Comparisons were made using the Mann-Whitney U test. Serum adiponectin, leptin, resistin and TNF- $\alpha$ were quantified using ELISA kits (Fujirebio, Tokyo, Japan). Liver fat percentage was assessed microscopically by biopsy samples.
Table I. Background characteristics of the patients.

\begin{tabular}{lcc}
\hline & Non-obese & Obese \\
\hline Number (male/female) & $11(5 / 6)$ & $14(7 / 7)$ \\
Height $(\mathrm{cm})$ & $164.7 \pm 8.6$ & $158.8 \pm 9.7$ \\
Weight $(\mathrm{kg})$ & $63.1 \pm 8.3$ & $76.8 \pm 9.2$ \\
BMI $\left(\mathrm{kg} / \mathrm{cm}^{2}\right)$ & $23.2 \pm 1.5$ & $30.6 \pm 4.0$ \\
ALT $(\mathrm{IU} / \mathrm{l})$ & $43.5 \pm 45.5$ & $68.9 \pm 71.2$ \\
TC $(\mathrm{mg} / \mathrm{dl})$ & $202.7 \pm 50.2$ & $185.1 \pm 42.4$ \\
TG $(\mathrm{mg} / \mathrm{dl})$ & $146.7 \pm 144.4$ & $116.4 \pm 52.6$ \\
FBS $(\mathrm{mg} / \mathrm{dl})$ & $103.4 \pm 27.4$ & $101.9 \pm 26.8$ \\
IRI $(\mu \mathrm{U} / \mathrm{ml})$ & $7.18 \pm 5.88$ & $18.10 \pm 17.08$ \\
HOMA-IR & $1.83 \pm 1.88$ & $5.09 \pm 5.74$ \\
Liver fat $(\%)$ & $30.45 \pm 21.62$ & $41.79 \pm 18.77$ \\
\hline
\end{tabular}

BMI, body mass index; ALT, alanine aminotransferase; TC, total cholesterol; TG, triglyceride; FBS, fasting blood sugar; IRI, immunoreactive insulin and HOMA-IR, homeostasis model assessment-insulin resistance.

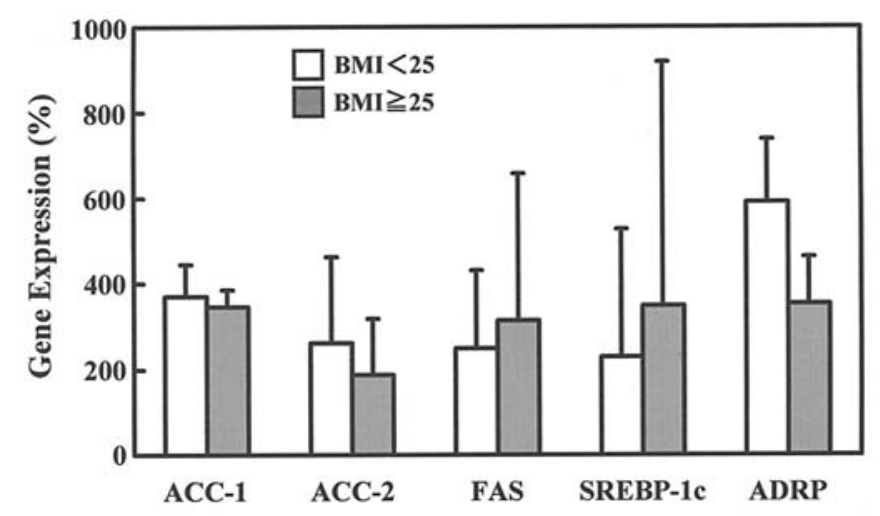

Figure 1. Real-time RT-PCR analysis of genes related to de novo synthesis and uptake of fatty acids in NAFLD patients with or without obesity. BMI, body mass index; ACC, acetyl-CoA carboxylase; FAS, fatty acid synthase; SREBP-1c, sterol regulatory element-binding protein 1c and ADRP, adipose differentiation-related protein (adipophilin).

\section{Results}

The expression of genes related to de novo synthesis and uptake of fatty acids. The process of fatty acid synthesis is promoted by acetyl-CoA carboxylase (ACC) -1, -2, and fatty acid synthase (FAS) (11). Both ACC and FAS were positively regulated by a transcriptional factor, sterol regulatory elementbinding protein 1c (SREBP 1c) (11). Fatty acids in hepatocytes are transferred from serum by adipose differentiation-related protein (ADRP) (12). The expression levels of all these genes were 2 - to 5-fold higher in NAFLD than in normal liver $(100 \%)$; however, the levels were not significantly different between the obese and non-obese groups (Fig. 1).

Expression of genes related to mitochondrial $\beta$-oxidation. Carnitine palmitoyltransferase 1a (CPT1a), which is a regulatory enzyme in mitochondrial $\beta$-oxidation, transfers 

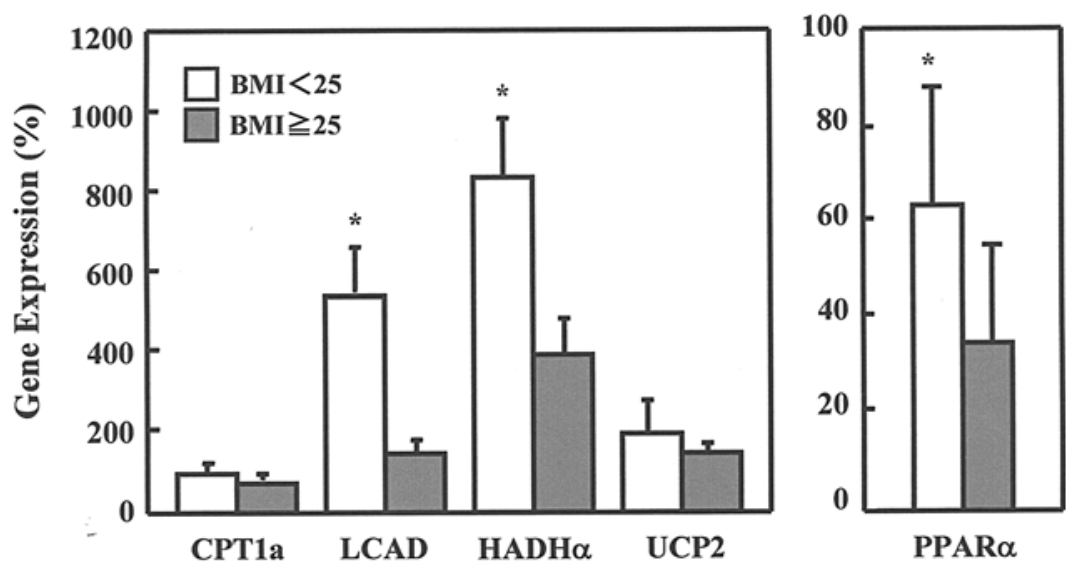

Figure 2. Real-time RT-PCR analysis of genes related to mitochondrial ß-oxidation in NAFLD patients with or without obesity. ${ }^{*}$ Significant difference (p<0.05) between the obese and non-obese groups. BMI, body mass index; CPT1a, carnitine palmitoyltransferase 1a; LCAD, long-chain acyl-CoA dehydrogenase; $\mathrm{HADH} \alpha$, long-chain L-3-hydroxyacyl-coenzyme A dehydrogenase $\alpha$; UCP2, uncoupling protein 2 and PPAR $\alpha$, peroxisome proliferators-activated receptor $\alpha$.

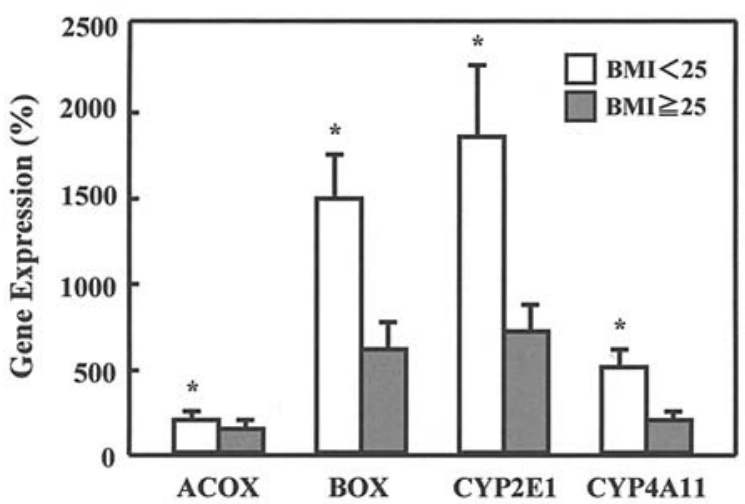

Figure 3. Real-time RT-PCR analysis of genes related to peroxisomal or microsomal oxidation in NAFLD patients with or without obesity. *Significant difference $(\mathrm{p}<0.05)$ between the obese and non-obese groups. BMI, body mass index; ACOX, straight-chain acyl-CoA oxidase; BOX, branched-chain acyl-CoA oxidase and CYP, cytochrome P450.

fatty acids from the cytosol to mitochondria. $\beta$-oxidation is then catalyzed by enzymes such as long-chain acyl-CoA dehydrogenase (LCAD) and long-chain L-3-hydroxyacylcoenzyme A dehydrogenase $\alpha(\mathrm{HADH} \alpha)$. Uncoupling protein 2 (UCP2), a mitochondrial inner-membrane protein emerging as a potential regulator of mitochondrial ROS production, mediates proton leak across the inner membrane and uncouples fuel oxidation from ATP synthesis $(13,14)$. Peroxisome proliferators-activated receptor $\alpha(\operatorname{PPAR} \alpha)$ is a major transcriptional activator of genes involved in mitochondrial B-oxidation, peroxisomal straight-chain acyl-CoA oxidase (ACOX) and microsomal CYP4As $(18,21)$. PPAR $\alpha$ also induces the expression of enzymes related to $ß$-oxidation (15-17). In NAFLD, CPT1a expression was almost the same as those in control $(100 \%)$ and no difference was seen between the obese and non-obese patients (Fig. 2). LCAD and HADH $\alpha$ levels were increased in NAFLD liver compared with normal controls and the increase was significantly greater in the non-obese patients ( $>5$ - and 8-fold for LCAD and HADH $\alpha$, respectively) than in obese patients (nearly 1.5- and 4-fold for LCAD and HADH $\alpha$, respectively) (Fig. 2). In NAFLD, UCP2 expression was nearly 2-fold higher compared with that in normal liver, with similar levels in the obese and non-obese groups (Fig. 2). In contrast, PPAR $\alpha$ expression was lower in NAFLD than the normal controls and the magnitude of suppression was significantly greater in obese patients (Fig. 2).

Expression of other genes related to fatty acid oxidation. When mitochondrial oxidative capacity is impaired, alternative oxidation pathways are activated in peroxisomes (ß-oxidation) and microsomes ( $\omega$-oxidation). ACOX and branched-chain acyl-CoA oxidase (BOX) are involved in peroxisomal $B$ oxidation (18). In microsomal $\omega$-oxidation, CYP2E1 and CYP4A11, and inducible hepatic microsomal cytochromes $\mathrm{P}-450$, initiate the autopropagative process of lipid oxidation (15-17). Therefore, the expression levels of these genes were examined (Fig. 3). All the genes tested, especially BOX, CYP2E1 and CYP4A11, were extensively up-regulated in NAFLD in comparison with the levels in normal controls $(100 \%)$. Moreover, the increasing ratio was significantly higher in non-obese patients.

Expression of genes related to antioxidant pathways. Molecules collectively referred to as ROS are formed in the fatty acid oxidation pathway. Cytotoxic ROS is normally catalyzed by antioxidant molecules such as superoxide dismutase (SOD), catalase, and glutathione, which is produced by glutathione synthetase (GSS) (19). In NAFLD, the expression of SOD and catalase were 5- and 10-fold higher, respectively, than in normal livers, whereas GSS expression was unchanged (Fig. 4). SOD and catalase levels were significantly higher in non-obese than obese patients (Fig. 4).

Expression of genes related to lipid droplet formation. Fatty acids are also metabolized into triglycerides by esterification and stored within lipid droplets. Diacylglycerol O-acyltransferase 1 (DGAT1) is involved in triglyceride synthesis and hormone sensitive lipase (HSL) is a lipolytic enzyme for catalyzing triglyceride accumulation. DGAT1 expression in NAFLD was slightly increased compared with normal liver (100\%), while HSL levels were decreased by $80 \%$ compared with normal liver (Fig. 4). No significant difference was seen 

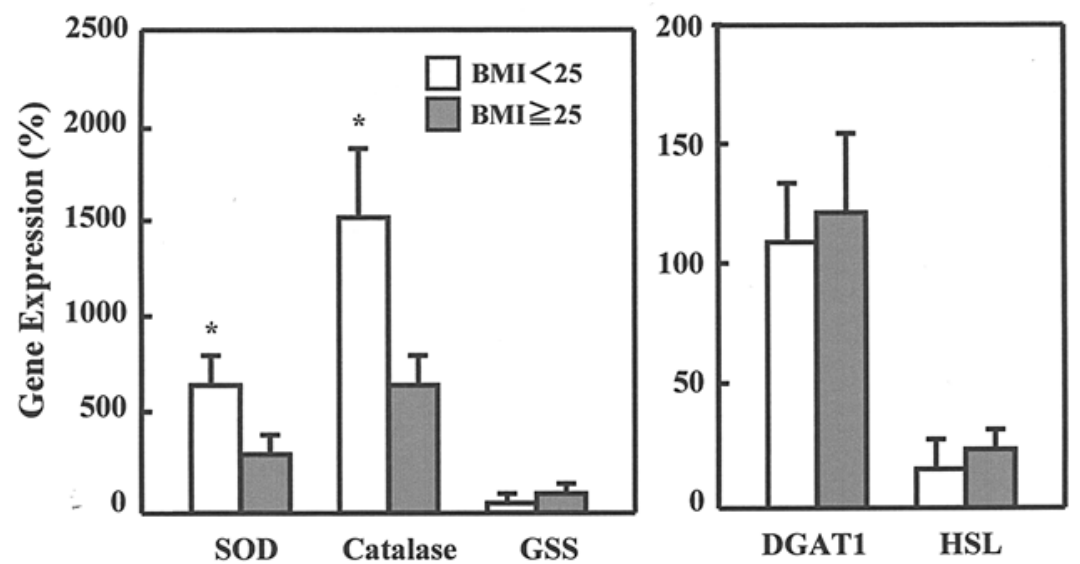

Figure 4. Real-time RT-PCR analysis of genes related to ROS elimination, triglyceride synthesis and lipolysis in NAFLD patients with or without obesity. "Significant difference $(\mathrm{p}<0.05)$ between the obese and non-obese groups. BMI, body mass index; SOD, superoxide dismutase; GSS, glutathione synthetase; DGAT1, diacylglycerol O-acyltransferase 1 and HSL, hormone sensitive lipase.

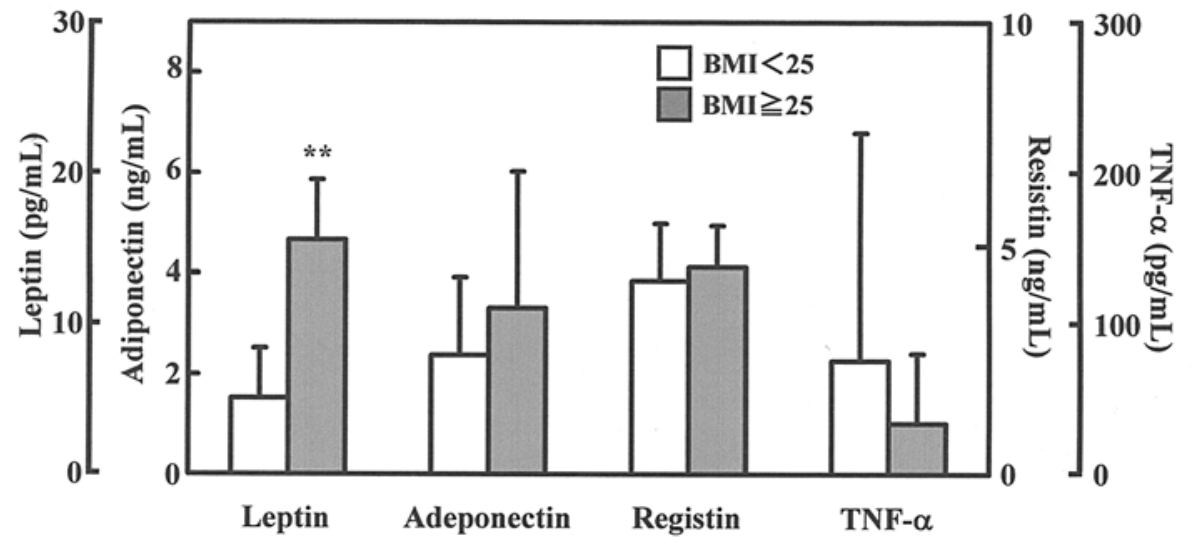

Figure 5. Serum adipocytokine levels in NAFLD patients with or without obesity. ${ }^{*}$ Significant difference $(\mathrm{p}<0.05)$ between the obese and non-obese groups. BMI, body mass index and TNF, tumor necrosis factor.

in either of the genes between the non-obese and obese groups (Fig. 4).

Serum levels of adipocytokines. Adipocytokines are important humoral factors that are associated with lipid and glucose metabolism. In contrast with the non-obese patients, insulinresistance was obvious in the obese patients (Table I). Therefore, we examined the serum values of the adipocytokines, leptin, adiponectin, resistin and TNF- $\alpha$. We found that, although there were no significant differences in adiponectin, resistin and TNF- $\alpha$ levels, leptin levels were significantly higher in obese patients (Fig. 5).

\section{Discussion}

Fatty acid metabolism in hepatocytes can occur via four mechanisms: a) de novo fatty acid synthesis and uptake of plasma-free fatty acids; b) fatty acid catalysis by mitochondrial, peroxisomal and microsomal oxidation; c) neutralization of ROS derived from fatty acid oxidation; and d) conversion between fatty acids and triglyceride. In this study, we investigated the differences in fatty acid metabolism between obese and non-obese NAFLD patients. Fatty acid metabolismrelated genes were generally overexpressed in NAFLD; however, the expression of genes associated with pathways of fatty acid synthesis and uptake, triglyceride synthesis and lipolysis was similar in the obese and non-obese groups (Figs. 1 and 4). An obvious difference was found in the fatty acid oxidation and ROS elimination pathways (Figs. 2, 3 and 4), therefore, the expression of the genes tested was significantly higher in the non-obese group.

These results indicate that, in obese patients, an overload of mitochondrial ß-oxidation, following fatty acid accumulation by up-regulation of de novo synthesis and uptake, cannot be compensated by $\beta$ - and $\omega$-oxidation in peroxisomes and microsomes, respectively. Nevertheless, this overload may, to some degree, be compensated in non-obese patients. This difference may result in more severe liver injury, insulin resistance and steatosis in obese patients compared with non-obese patients, as shown in Table I. What derives the difference in fatty acid metabolism between the obese and non-obese groups? At present, there are no clear answers; however, one clue may be the serum levels of adipocytokines. Although serum levels of some adipocytokines are strongly 
affected by gender, there was no disparity in gender between the obese and non-obese groups. Here, we found that only leptin levels were markedly higher in the obese group (Fig. 5).

Leptin is a hormone produced by adipocytes and exerts metabolic effects in peripheral tissues, for example, leptin induces PPAR $\alpha$ gene expression and stimulates fatty acid oxidation (20). As a consequence of their increased fat mass, elevated circulating levels of leptin in obese patients is to be expected. The expression of leptin receptors was also upregulated in NAFLD patients but the levels were not different between the obese and non-obese patients (data not shown). Therefore, if leptin and its signaling pathway functioned normally in obese patients, the expression of PPAR $\alpha$ and other genes related to fatty acid oxidation should be more upregulated in obese than in non-obese patients. The unexpected response of fatty acid oxidation-associated genes and PPAR $\alpha$ in our study may thus be explained by leptin resistance (21). The mechanism of leptin resistance still remains unclear. Because the peripheral effect of leptin is, in part, through the central nervous system, defective leptin transport across the blood-brain barrier may be implicated in this process (22). Many institutes have begun to examine the mechanisms of leptin receptor signal attenuation $(23,24)$. They mainly investigated the inhibitory molecules for leptin receptor signaling such as suppressor of cytokine signaling-3 (SOCS-3). We also tested the expression of SOCS-3; however, the expression levels were suppressed in both the obese and nonobese groups (data not shown). Therefore, other factors associated with under-responsiveness to leptin should be evaluated in our patients.

In summary, our results indicate the following: i) Fatty acid synthesis and uptake were increased similarly in both the obese and non-obese groups, despite an accumulation of fatty acids in hepatocyte. ii) In obese patients, fatty acid oxidation and ROS elimination was insufficiently compensated, which resulted in worse liver damage, steatosis and insulin resistance in these patients. iii) Differences in fatty acid metabolism between the obese and non-obese groups might be due to leptin resistance. Further studies are needed to clarify the relationship between leptin resistance and NAFLD.

\section{References}

1. Browning JD and Horton JD: Molecular mediators of hepatic steatosis and liver injury. J Clin Invest 114: 147-152, 2004.

2. Clark JM, Brancati FL and Diehl AM: The prevalence and etiology of elevated aminotransferase levels in the United States. Am J Gastroenterol 98: 960-967, 2003.

3. Angulo P: Nonalcoholic fatty liver disease. N Engl J Med 346: 1221-1231, 2002.

4. Ludwig J, Viggiano TR, McGill DB and Oh BJ: Nonalcoholic steatohepatitis: Mayo Clinic experiences with a hitherto unnamed disease. Mayo Clin Proc 55: 434-438, 1980.

5. Matteoni CA, Younossi ZM, Gramlich T, Boparai N, Liu YC and McCullough AJ: Nonalcoholic fatty liver disease: a spectrum of clinical and pathological severity. Gastroenterology 116: 1413-1419, 1999.

6. Nakamuta M, Kohjima M, Morizono S, Kotoh K, Yoshimoto T, Miyagi I and Enjoji M: Evaluation of fatty acid metabolismrelated gene expression in nonalcoholic fatty liver disease. Int J Mol Med 16: 631-635, 2005.
7. Kohjima M, Enjoji M, Higuchi N, Katoh M, Kotoh K, Yoshimoto T, Fujino T, Yada M, Yada R, Harada N, Takayanagi $\mathrm{R}$ and Nakamuta M: Re-evaluation of fatty acid metabolism-related gene expression in nonalcoholic fatty liver disease. Int J Mol Med 20: 351-358, 2007.

8. Kohjima M, Higuchi N, Katoh M, Kotoh K, Yoshimoto T, Fujino T, Yada M, Yada R, Harada N, Enjoji M, Takayanagi R and Nakamuta M: SREBP-1c, regulated by the insulin and AMPK signaling pathways, plays a role in nonalcoholic fatty liver disease. Int J Mol Med 21: 507-511, 2008.

9. Kato M, Higuchi N and Enjoji M: Reduced expression of ATGL and CGI-58 in the liver may attribute to develop NAFLD in patients with insulin resistant background. Scand J Gastroenterol (In press).

10. Kato M, Higuchi N, Syundo N, Kotoh K, Kohjima M, Nakamuta M, Takayanagi R and Enjoji M: LXR works in cooperation with SREBP-1c as a major regulator of lipid synthesis in the liver with NAFLD. Hepatol Res (In press).

11. Horton JD, Goldstein JL and Brown MS: SREBPs: activators of the complete program of cholesterol and fatty acid synthesis in the liver. J Clin Invest 109: 1125-1131, 2002.

12. Chang BH, Li L, Paul A, Taniguchi S, Nannegari V, Heird WC and Chan L: Protection against fatty liver but normal adipogenesis in mice lacking adipose differentiation-related protein. Mol Cell Biol 26: 1063-1076, 2006.

13. Negre-Salvayre A, Hirtz C, Carrera G, Cazenave R, Troly M, Salvayre R, Penicaud L and Casteilla L: A role for uncoupling protein-2 as a regulator of mitochondrial hydrogen peroxide generation. FASEB J 11: 809-815, 1997.

14. Krauss S, Zhang CY and Lowell BB: A significant portion of mitochondrial proton leak in intact thymocytes depends on expression of UCP2. Proc Natl Acad Sci USA 99: 118-122, 2002.

15. Yu S, Rao S and Reddy JK: Peroxisome proliferator-activated receptors, fatty acid oxidation, steatohepatitis and hepatocarcinogenesis. Curr Mol Med 3: 561-572, 2003.

16. Reddy JK and Hashimoto T: Peroxisomal beta-oxidation and peroxisome proliferator-activated receptor alpha: an adaptive metabolic system. Annu Rev Nutr 21: 193-230, 2001.

17. Reddy JK: Nonalcoholic steatosis and steatohepatitis. III. Peroxisomal beta-oxidation, PPAR alpha, and steatohepatitis. Am J Physiol Gastrointest Liver Physiol 281: G1333-G1339, 2001.

18. Wanders RJ: Peroxisomes, lipid metabolism, and peroxisomal disorders. Mol Genet Metab 83: 16-27, 2004.

19. Pessayre D, Fromenty B and Mansouri A: Mitochondrial injury in steatohepatitis. Eur J Gastroenterol Hepatol 16: 1095-1105, 2004.

20. Suzuki A, Okamoto S, Lee S, Saito K, Shiuchi T and Minokoshi Y: Leptin stimulates fatty acid oxidation and peroxisome proliferators-activated receptor $\alpha$ gene expression in mouse $\mathrm{C} 2 \mathrm{C} 12$ myoblasts by changing the subcellular localization of the $\alpha 2$ form of AMP-activated protein kinase. Mol Cell Biol 27: 4317-4327, 2007.

21. Münzberg H, Björnholm M, Bates SH and Myers MG Jr: Leptin receptor action and mechanisms of leptin resistance. Cell Mol Life Sci 62: 642-652, 2005.

22. Kastin AJ, Pan W, Maness LM, Koletsky RJ and Ernaberger P: Decreased transport of leptin across the blood-brain barrier in rats lacking the short form of the leptin receptor. Peptide 20: 1449-1453, 1999.

23. Bjorbaek C, El Haschmi K, Frantz JD and Flier JS: The role of SOCS-3 in leptin signaling and leptin resistance. J Biol Chem 274: 30059-30065, 1999.

24. Kimura A, Kinjyo I, Matsumura Y, Mori H, Mashima R, Harada M, Chien KR, Yasukawa $\mathrm{H}$ and Yoshimura A: SOCS3 is a physiological negative regulator for granulopoiesis and granulocyte colony-stimulating factor receptor signaling. J Biol Chem 279: 6905-6910, 2004. 